\title{
FIB Preparation of Mesa Structures for SIMS Analysis
}

\author{
J. M. McKinley, B. B. Rossie, M. A. Decker, and *F. A. Stevie
}

Agere Systems, 9333 S. John Young Parkway, Orlando, FL 32836

*North Carolina State University, 1010 Main Campus Drive, Box 7531, Raleigh, NC 27695

Good depth resolution is one of the most important attributes of SIMS depth profiling. Gated detection of secondary ions from a small region within a larger raster is the method typically used to provide the desired depth resolution. The raster and gate approach reduces the area that must have a uniform depth, and minimizes the possibility of sidewall contributions.

One current challenge is the need to provide an analysis from a region that has often been subject to reduction in size without sacrificing depth resolution. On semiconductor wafers, the region between devices available for dedicated SIMS analysis patterns is now no larger than $80 \mu \mathrm{m}$ in one dimension. Therefore the SIMS crater for a depth profile must be on the order of $75 \mu \mathrm{m}$ or less. This task is often further complicated by the presence in regions adjacent to the analysis area of a high concentration of an element that must be detected at low concentration. One possibility is to perform this analysis without sidewalls. This can be achieved using sample preparation to remove most of the specimen, leaving only a mesa or pillar that is smaller than the ion beam raster. [1] This is a time intensive and risk prone process. Another approach would be a mask generation of the desired pattern, [2] but this would require etching that might be unique to the wafer and involve additional cost for each wafer. Contemporary FIB workstations have the accuracy and primary current density required to locally remove the sidewalls. Figure 1 shows an example of a FIB prepared SIMS analysis structure from a SIMS test pattern on a semiconductor wafer. The region on the left has been totally excavated to check for ion milling effects and the region on the right has been ion milled to provide a mesa structure. Typical overall milled dimensions were an $80 \mu \mathrm{m}$ square with different mesa sizes. Note that many of the semiconductor applications do not have requirements for deep craters, so that a depth of $1 \mu \mathrm{m}$ may be sufficient. Preparation for one site was achieved in approximately $0.5 \mathrm{hr}$ using a FEI 200TEM FIB. Figure 2 shows SIMS B and As depth profiles from a SIMS test pattern similar to Fig.1 with and without the FIB removed region. SIMS analyses were obtained using a CAMECA IMS-6f with $\mathrm{Cs}^{+}$primary beam and $30 \mu \mathrm{m}$ diameter detected area. No difference was noted with a $60 \mu \mathrm{m}$ raster, but the figure shows an improvement in the As profile for the FIB milled pillar when the raster was reduced to $40 \mu \mathrm{m}$ (nominal raster size - actual size larger by 1 beam dia.).

The results show that the concept is practical but only limited improvement was achieved. Different configurations of the removed material need to be studied to optimize depth resolution and determine the true value of this approach. This method may also be useful to study an array of small areas, such as a particular segment of a semiconductor transistor, or particles surrounded by a material that may cause a mass interference or charging due to an insulating material.

[1] R. von Criegern, I Weitzel, and J. Fortner, Secondary Ion Mass Spectrometry, SIMS IV, edited by A. Benninghoven, J. Okano, R. Shimizu, and H. W. Werner, Springer, Berlin, 1984, 308.

[2] F. A. Stevie, J. Vac. Sci. Technol. B10 (1992) 323. 


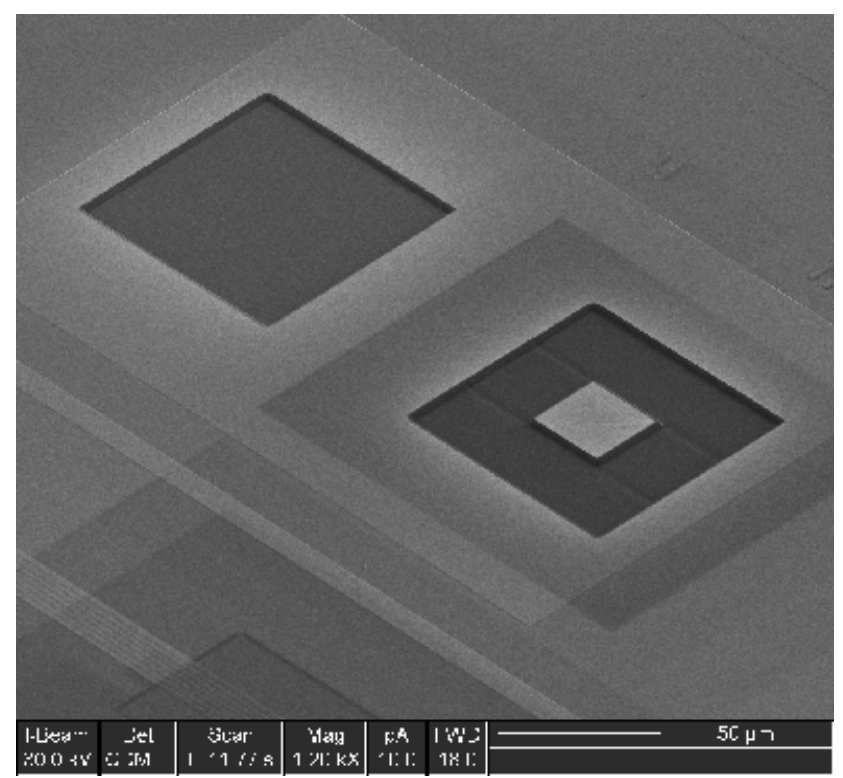

FIG.1 FIB secondary electron image of FIB milled structures

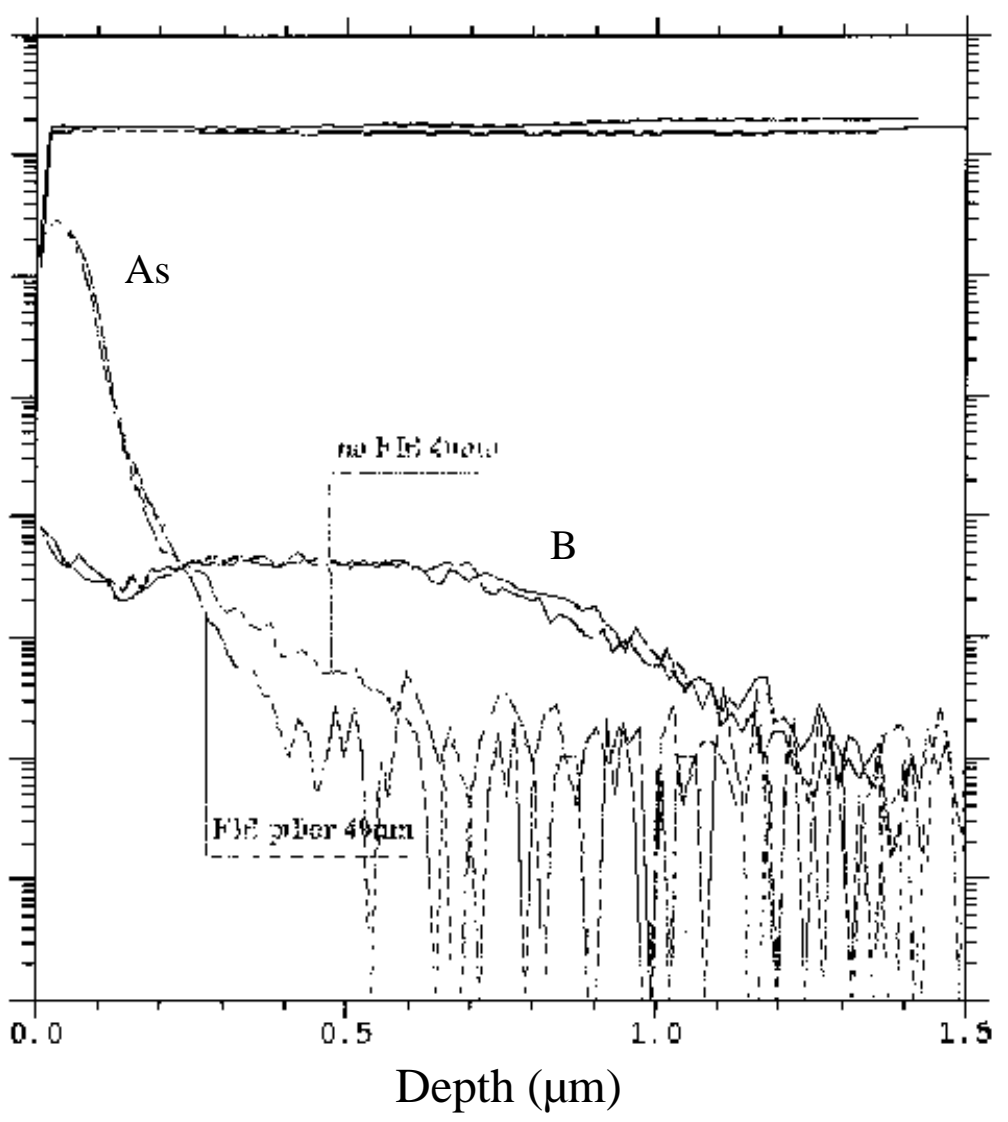

FIG. 2 SIMS depth profiles on regions with and without FIB pillar 